\title{
Suicide rates among patients subject to community treatment orders in England during 2009-2018
}

Isabelle M. Hunt, Roger T. Webb, Pauline Turnbull, Jane Graney, Saied Ibrahim, Jenny Shaw, Nav Kapur and Louis Appleby

\section{Background}

Community treatment orders (CTOS) enable patients to be treated in the community rather than under detention in hospital.

Population-based studies of suicide among patients subject to a CTO are scarce.

\section{Aims}

To compare suicide rates among patients subject to a CTO with all discharged psychiatric patients and those detained for treatment but not subject to a CTO at discharge ('CTO-eligible' patients).

\section{Method}

From a national case series of patients who died by suicide within 12 months of contact with mental health services in England during 2009-2018, we estimated average annual suicide rates for all discharged patients, those on a CTO at the time of suicide, those ever treated under a СТО and Сто-eligible patients.

\section{Results}

Suicide rates for patients on a СTO at the time of suicide (191.3 per 100000 patients) were lower than all discharged patients (482.3 per 100000 discharges). Suicide rates were similar in those ever treated under a CTO (350.1 per 100000 CTOs issued) and in CTO-eligible patients (382.9 per 100000 discharges).
Suicide rates within 12 months of discharge were higher in persons ever under a CTO (205.1 per 100000 CTOs issued) than CTOeligible patients (161.5 per 100000 discharges), but this difference was reversed for rates after 12 months of discharge (153.2 per 100000 CTOs issued v. 223.4 per 100000 discharges).

\section{Conclusions}

CTOs may be effective in reducing suicide risk. The relative benefits of CTOs and intensive aftercare may be time-dependent, with the benefit of a CTO being less before 12 months after discharge but greater thereafter. CTO utilisation requires a careful balancing of patient safety versus autonomy.

\section{Keywords}

Community treatment orders; suicide; psychiatric patients; mental health; mental health act.

\section{Copyright and usage}

(C) The Author(s), 2021. Published by Cambridge University Press on behalf of the Royal College of Psychiatrists. This is an Open Access article, distributed under the terms of the creative commons Attribution licence (https://creativecommons.org/ licenses/by/4.0/), which permits unrestricted re-use, distribution, and reproduction in any medium, provided the original work is properly cited.

\section{Background}

Suicide represents a global public health problem, with over 700000 people dying by suicide each year. ${ }^{1}$ According to the most recent data in England and Wales, in 2019, there were 5691 suicides registered, representing 11.0 deaths per 100000 population. ${ }^{2}$ Mental illness is strongly associated with an increased suicide risk ${ }^{3}$ and all member states of the World Health Organization (WHO) have committed to improving mental health and reaching a global target of reducing suicide rates by a third by $2030 .{ }^{4}$ Identifying high-risk groups is a key preventative goal, and people under the care of mental health services, including in-patients, have been identified as a priority in England's National Suicide Prevention Strategy. ${ }^{5}$ For those with severe mental illness with impaired decision-making capacity, the use of compulsory admission and detainment for treatment under the Mental Health Act 1983 (MHA; amended 2007) is considered where there is a potential risk of harm to the patient or others. Two common detaining sections of the MHA are Section 3 (admission for treatment) and Section 37 (hospital order for those convicted of a criminal offence).

The introduction of community treatment orders (CTOs) in England and Wales in 2008 provided the option for people detained under the MHA to be treated in the community rather than in hospital. Their purpose was to reduce levels of readmission and provide supervised treatment in a less restrictive environment, while maintaining close clinical contact. Two mandatory conditions attached to a CTO involve the availability of the patient for assessment at the time of CTO renewal, and agreement to be seen by a second psychiatrist if the individual is too unwell to consent to receiving drug treatment themselves. Discretionary conditions can also be applied with the patient's agreement, typically prescribed treatment adherence and service engagement. Although treatment is not coercively imposed on patients in the community, those who show deterioration in mental health may be recalled back to hospital for assessment and medication. Qualitative studies have indicated that patients benefit from closer involvement in their care planning under a CTO, ${ }^{6,7}$ and family caregivers have viewed CTOs as a 'safety net', welcoming the option of recall at times of relapse. ${ }^{8,9}$ However, the coercive nature of supervised community treatment has caused much debate, with critics viewing CTOs as punitive, restricting civil liberties ${ }^{10}$ and compromising trust in the patient-psychiatrist relationship. ${ }^{11}$ A recent systematic review and meta-analysis found inconsistent evidence that compulsory community treatment improved patient outcomes. ${ }^{12}$ It also confirmed findings from the only randomised controlled trial of CTOs in England, the Oxford Community Treatment Order Evaluation Trial (OCTET), that CTOs did not reduce rates of readmission. ${ }^{13,14}$ Other studies in England have reported patients on a CTO had more hospital readmissions and spent longer in in-patient care compared with those not on a CTO. ${ }^{15,16}$ Despite the sparse evidence on whether CTOs reduce bed use and improve treatment response, they are used extensively in many countries and are often initiated to potentially reduce the risk of self-harm and suicide after discharge. 


\section{CTOs and suicide}

The National Confidential Inquiry into Suicide and Safety in Mental Health (NCISH) ${ }^{17}$ found $126(1 \%)$ deaths by suicide among patients with recent mental health service contact were subjected to a CTO during 2009-2017, two-thirds of whom were on a CTO when they died. Of note, a third of patients subject to a CTO who died within 3 months of discharge were preceded by treatment non-adherence and missed service contact - two key elements of CTO provision. The evidence base for the effectiveness of CTOs in reducing suicide is limited: one UK observational controlled study found no association between CTOs and suicide risk among patients with schizophrenia, ${ }^{18}$ whereas a USA study found that mandated community treatment reduced suicide risk. ${ }^{19}$ Further knowledge is needed to enhance our understanding of the clinical profile of patients who are placed on a CTO who subsequently die by suicide. We utilised a national case series to examine suicide risk in patients who had been treated under a CTO. Our objectives were to calculate suicide rates among patients under a CTO, and to compare rates and features of patients subject to a CTO with those who were not under CTO but who were eligible to be so; i. e. they had been detained for treatment under Section 3 or 37 of the MHA at their last admission. For this comparison, we examined suicides among patients who had ever been under CTO after hospital discharge, to take into account the possibility that the period at risk for patients dying when under a CTO may have been shorter than the equivalent period for those who were eligible for a CTO. We hypothesised that suicide risk would be highest among patients subject to a CTO (on the grounds that management without legal restriction had been viewed by clinicians as inadequate), followed by CTO-eligible patients and then all other patients discharged from in-patient psychiatric care.

\section{Method}

\section{Study data-set}

We used data from the NCISH. A detailed description of NCISH methodology is described elsewhere. ${ }^{20}$ In brief, there are three stages to data collection. First, NCISH is notified from the Office for National Statistics (http://wwww.ons.gov.uk) of all unnatural deaths in England with a coronial verdict of suicide or of undetermined intent ('open' verdict). Second, details of the deceased are sent to mental health services in the individual's residential district to identify those who had been in service contact in the 12 months before death ('patient suicides'). Third, sociodemographic and clinical data on patient suicides are collected via a questionnaire completed by the responsible clinician. The questionnaire collects detailed information on patients who died by suicide in the community, including whether the patient was on a CTO at the time of their last discharge or death, and the length of time spent under a CTO ( $<6$ months, between 6 months and 1 year, and $\geq 1$ year). In addition, the nature of patients' previous admissions (voluntary, detained for assessment or treatment, under the power of recall of a CTO) were recorded.

Deaths that were assigned an open verdict were included in the study, as is conventional in suicide research in the UK, ${ }^{21}$ and are hereon referred to as 'suicides' in this paper. We selected all patients who died by suicide during years 2009 and 2018, inclusive.

\section{Denominator data}

For overall suicide rates among discharged patients, we applied the number of discharges as the denominator, obtained from the Mental Health Services Data Set (MHSDS) via NHS Digital. ${ }^{22}$ This national data-set holds patient-level data on all individuals in contact with in-patient, out-patient and community mental health services. To calculate suicide rates among patients who were ever subject to a CTO, we applied the number of open CTOs for the years $2009 / 10$ to $2018 / 19$ as the denominator. We also calculated an overall suicide rate among patients who were on a CTO at the time of death, using the denominator figures on 31 March to give a typical rate on any one day (MHSDS data were available from 2010 onward). Suicide rates among CTO-eligible patients (i.e. those detained for treatment under Section 3 or Section 37 of the MHA, but who were discharged to voluntary community care) were calculated per 100000 admissions under Section 3 or Section 37 (excluding discharges from a CTO), with MHSDS data available for the years 2011-2018. Denominator data to calculate these rates included conversions to a Section 3 or Section 37 for those who had been voluntarily admitted. The numbers of admissions, discharges and CTOs issued were available by financial year initially; we then converted them to being by calendar year, for consistency with the numerators.

\section{Statistical analysis}

We first calculated suicide rates (and their 95\% confidence intervals) across the entire observation period for all patients discharged from psychiatric care, as a baseline against which suicide rates in the subgroups of interest could be compared. Suicide rates among patients ever subject to a CTO were calculated by the total number of CTOs issued; suicide rates among patients on a CTO at the time of death were calculated by the total number of patients on a CTO on the 31 March of each year. Suicide rates among CTOeligible patients were compared with those for persons ever treated under a CTO, stratified according to whether they had died within or after 12 months of discharge. Calendar year was fitted as a continuous variable in a Poisson regression model, to test for linear temporal trends in the suicide rate. ${ }^{23}$ Poisson models were tested for evidence of significant overdispersion, using the likelihood ratio test of $a=0$ that is generated by the negative binomial model. Where tests indicated inadequate goodness of fit, the parameters from the negative binomial model were reported instead of those from the Poisson model. ${ }^{23}$ Because of small numerator values, we collapsed years into categories of 2011-2014 and 20152018 and compared rates between patients subject to a CTO and CTO-eligible patients via incidence rate ratios (IRRs) and their exact $95 \%$ confidence intervals. Comparison of patient characteristics between those on a CTO and all other patients (i.e. those not treated under a CTO) were conducted with chi-squared tests, with statistical significance indicated at the two-sided 5\% level.

Over the study's observation period, the overall response rate for questionnaires was 95\%, but was lower during calendar years 2016 (94\%), 2017 (90\%) and 2018 (71\%) because of the time associated with legal processes and data collection. We therefore estimated the number of suicides in 2016-2018, based on the expected final return of questionnaires for the previous 7 years (2009-2015), to enable comparable numerator data when assessing rates across the study period. Descriptive statistics of patient characteristics were carried out on actual cases. All statistical analysis was performed with Stata software version 15.1 for Windows. ${ }^{24}$ Ethical approval was obtained from the North West - Greater Manchester South Research Ethics Committee (reference: ERP/96/136). NCISH also has Section 251 approval under the NHS Act 2006 (reference: PIAG 4-08(d)/2003).

\section{Results}

Over the study period 2009-2018, there were 12771 suicides by people who had been in contact with mental health services in the 
Table 1 Suicide rates among all discharged patients and among patients on a CTO when they died

\begin{tabular}{|c|c|c|c|c|c|c|c|c|c|}
\hline \multirow[b]{2}{*}{ Period of observation, years } & \multicolumn{3}{|c|}{ All discharged patients } & \multicolumn{3}{|c|}{ Patients on a Сто at date of death } & \multicolumn{3}{|c|}{ Relative risks: IRRs } \\
\hline & $n$ & Rate $^{a}$ & $95 \% \mathrm{Cl}$ & $n$ & Rate $^{b}$ & $95 \% \mathrm{Cl}$ & IRR & $95 \% \mathrm{Cl}$ & $P$-value \\
\hline 2009-2013 & 3052 & 515.4 & $497.4-534.0$ & $37^{\mathrm{c}}$ & 210.3 & $148.1-289.7$ & 0.41 & $0.29-0.56$ & $<0.001$ \\
\hline 2014-2018 & 2641 & 449.0 & $432.1-466.4$ & 48 & 178.8 & 131.9-237.0 & 0.40 & $0.29-0.53$ & $<0.001$ \\
\hline Total & 5693 & 482.3 & $469.9-495.0$ & 85 & 191.3 & $152.8-236.4$ & 0.40 & $0.32-0.49$ & $<0.001$ \\
\hline \multicolumn{10}{|c|}{$\begin{array}{l}\text { CTO, community treatment order; IRR, incidence rate ratio. } \\
\text { a. Rate per } 100000 \text { discharges from in-patient care. } \\
\text { b. Rate per } 100000 \text { patients subject to a CTO on the } 31 \text { March of each year. } \\
\text { c. Data shown for } 2010-2013 \text { as denominator incomplete in } 2009 \text {, therefore the total does not total } 93 \text { as reported in the main text. }\end{array}$} \\
\hline
\end{tabular}

12 months before they died. Of these, 11921 (93\%) were patients treated in the community, and 143 were treated under a CTO at their last discharge (referred to as 'ever subject to a CTO' herein); $3.9 \%$ of all community patient suicides with a previous admission, or $1.2 \%$ of all community patient suicides. This represents a mean of 14 suicide deaths per year in those ever subject to a CTO. At date of death, 93 patients were still on a CTO and 47 had had their CTO revoked; in the remaining three cases, CTO status at date of death by suicide was unknown. There were 483 CTO-eligible patients during the observation period, i.e. those who had been detained for treatment but were not under a CTO at discharge. This group represented $7.4 \%$ of all community patient suicides with a previous admission, or $4.1 \%$ of all community patient suicides. Suicide rates among CTO-eligible patients were calculated for those who died during 2011-2018 $(n=377)$ as denominator data were available for that period only.

\section{Characteristics of patient suicides ever treated under a CTO}

The median age of patients ever subject to a CTO $(N=137)$ was 44 years (interquartile range 16 years) and 85 out of 137 (62\%) were male. Over a quarter (39 out of $137,28 \%$ ) were from a minority ethnic group, proportionally more than other patients (832 out of $10599,8 \% ; P<0.001)$. The suicide method profile of the CTO group differed from that of other patient suicides, with a higher proportion jumping from a height or in front of a moving vehicle (46 out of $136,34 \%$ v. 1492 out of $10851,14 \% ; P<0.001$ ) and a lower proportion of self-hangings/strangulations (42 out of 136 , $31 \%$ v. 4974 out of $10851,46 \%$; $P=0.001$ ). The most common primary psychiatric diagnosis was schizophrenia and other delusional disorders (108 out of $137,79 \%$ v. 1536 out of $10688,14 \%$ of other patients; $P<0.001)$. Patients subject to a CTO more often had coexisting drug dependence or misuse (37 out of 137, 27\% v. 1075 out of $10633,10 \% ; P<0.001)$.

\section{Suicide rates}

Between 2010 and 2018, the overall suicide rate among patients who were under a CTO at date of death was 191.3 per 100000 CTO patients on the 31 March (Table 1). There was no overall linear temporal trend in rates across the study period (linear trend; 1 d.f. = 1.08; $P=0.30$; see Supplementary Fig. 1 available at https://doi. org/10.1192/bjo.2021.1021). Suicide rates among patients under a CTO were lower compared with all patients discharged from in-patient care at 482.3 per 100000 discharges (IRR 0.40; 95\% CI $0.32-0.49 ; P<0.001$ ) (Table 1). There was a fall in the number (linear trend; 1 d.f. $=8.5 ; P=0.004$ ) and rate (linear trend; 1 d.f. $=$ 4.22; $P=0.04)$ of suicide among discharged patients across the observation period (Supplementary Fig. 1).

Table 2 shows the numbers and rates of suicide among patients ever subject to a CTO and among CTO-eligible patients (i.e. those detained for treatment but were not under a CTO at discharge). Although the number of CTOs issued increased across the observation period, the number and rate of suicide fluctuated, and there was no evidence of linear temporal trend ( 1 d.f. $=0.07 ; P=0.79$ ). There was, however, a significant increase in the rate of suicide in CTO-eligible patients between 2011 and 2018 (linear trend; 1 d.f. $=11.38 ; P=0.001$ ).

Annual suicide rates were significantly lower among patients on a CTO at the time of death (Table 1) compared with those ever subject to a CTO (350.1 per 100000 CTOs issued; IRR 0.55; 95\% CI $0.44-0.68 ; P<0.001)$ and CTO-eligible patients (382.9 per 100 000 discharges; IRR 0.50; 95\% CI 0.40-0.62; $P<0.001)$ ) (Table 2). The suicide rate for CTO-eligible patients was slightly higher than for those ever treated under a CTO, but this difference did not reach statistical significance (IRR 1.09; 95\% CI 0.99-1.21; $P=$ 0.09). However, the rate among CTO-eligible patients who died within 12 months of discharge was lower than for patients ever subject to a CTO who died within 12 months of discharge (161.5 v. 205.1; $P=0.045$; Table 3 ). The opposite was found when comparing suicide rates after 12 months of discharge (Table 4), when rates in patients ever subject to a CTO were lower than among patients who were CTO-eligible (153.2 v. 223.4; $P=0.003)$.

\section{Discussion}

Our results suggest that suicide under CTO is a rare event, accounting for around $1 \%$ of all psychiatric patient suicides that occur in the community in England, for an average of 14 deaths per year nationally. We found a lower suicide rate among patients who were under a CTO when they died, compared with the rate among all discharged patients. Suicide rates were significantly lower for patients under a CTO at death compared with both the rate in patients ever

\begin{tabular}{|c|c|c|c|c|c|c|c|c|c|}
\hline \multirow[b]{2}{*}{ Period of observation, years } & \multicolumn{3}{|c|}{ Ever placed on a СТО } & \multicolumn{3}{|c|}{ Сто-eligible patients } & \multicolumn{3}{|c|}{ Relative risks: IRRS } \\
\hline & $n$ & Rate $^{a}$ & $95 \% \mathrm{Cl}$ & $n$ & Rate $^{b}$ & $95 \% \mathrm{Cl}$ & IRR & $95 \% \mathrm{Cl}$ & $P$-value \\
\hline 2011-2014 & 74 & 418.5 & $328.7-525.1$ & 172 & 325.9 & $279.1-378.4$ & 0.78 & $0.67-0.90$ & 0.001 \\
\hline 2015-2018 & 54 & 286.0 & 214.9-373.0 & 205 & 448.6 & $389.4-514.3$ & 1.57 & $1.36-1.80$ & $<0.001$ \\
\hline Total & 128 & 350.1 & $292.1-416.1$ & 377 & 382.9 & $345.3-423.5$ & 1.09 & $0.99-1.21$ & 0.09 \\
\hline
\end{tabular}


Table 3 Suicide rates among patients ever placed on a CTO and among СTO-eligible patients who died within 12 months of discharge

\begin{tabular}{|c|c|c|c|c|c|c|c|c|c|}
\hline \multirow[b]{2}{*}{ Period of observation, years } & \multicolumn{3}{|c|}{ Ever placed on a СТО } & \multicolumn{3}{|c|}{ CTO-eligible patients } & \multicolumn{3}{|c|}{ Relative risks: IRRS } \\
\hline & $n$ & Rate $^{a}$ & $95 \% \mathrm{Cl}$ & $n$ & Rate $^{b}$ & $95 \% \mathrm{Cl}$ & IRR & $95 \% \mathrm{Cl}$ & $P$-value \\
\hline 2011-2014 & 48 & 271.5 & $200.2-359.7$ & 72 & 136.4 & $106.8-171.8$ & 1.99 & $1.47-2.64$ & $<0.001$ \\
\hline 2015-2018 & 27 & 143.0 & $94.3-208.0$ & 87 & 190.4 & $152.5-234.8$ & 0.75 & $0.49-1.09$ & 0.13 \\
\hline Total & 75 & 205.1 & $161.4-257.1$ & 159 & 161.5 & 137.4-188.6 & 1.27 & $1.00-1.59$ & 0.045 \\
\hline $\begin{array}{l}\text { CTO, community treatment order; } \\
\text { a. Rate per } 100000 \text { CTOs issued. } \\
\text { b. Rate per } 100000 \text { discharges. }\end{array}$ & & & & & & & & & \\
\hline
\end{tabular}

subject to a CTO and in CTO-eligible patients. Overall, the suicide rate among patients ever treated under a CTO was not significantly different from the rate among patients who were CTO-eligible. We also found a difference in rates in relation to time under a CTO, with higher suicide rates within 12 months of discharge among all patients subject to a CTO compared with those who were CTO-eligible. However, after 12 months of discharge, this difference reversed, with the suicide rate among patients subject to a CTO becoming significantly lower than the rate among CTO-eligible patients.

It is well-established that suicide risk is greatly elevated after discharge from in-patient psychiatric care. ${ }^{25,26}$ However, both patients under a CTO and those eligible for a CTO appear to be at lower suicide risk compared with all discharged patients. There are two reasons to consider that our findings may suggest a benefit from CTOs specifically. First, the CTO-eligible patients are likely to have been at lower risk than the CTO group, hence the decision not to treat under a CTO, and the comparison of suicide rates should take into account this possible difference in risk. Second, the lower suicide rate in those under a current CTO suggests that an active CTO confers most protection. Our finding of a higher suicide rate among patients subject to a CTO within 12 months of discharge, and a lower rate more than a year after discharge, may be an indication that clinical stability takes longer to establish for patients subject to a CTO and that the main benefit is seen long term. Although there may be a causal effect that is attributable to improved care, alternative explanations are also possible, such as a longer period of pre-discharge stability in patients preparing for CTO, or selection of patients whose illnesses are more responsive to treatment.

Lower all-cause mortality rates have been previously reported in patients subject to a CTO compared with control patients, with closer community follow-up and opportunity for early identification of physical health problems cited as possible explanations. ${ }^{15,27}$ Such findings provide support for the effectiveness of CTOs in providing intensive case management in the community. Other studies have also suggested that patients under CTO have better engagement with community teams and sustained contact with services compared with non-CTO groups, ${ }^{28,29}$ and published evidence has shown that optimising continuity of care for vulnerable patients is effective in reducing suicide risk. ${ }^{30}$

Our findings concur with those reported from previous studies, which showed that patients under a CTO have more severe mental illness and are younger compared with those discharged without a CTO. ${ }^{15,29}$ Over a quarter of the patients subject to a CTO who died by suicide in our study belonged to an ethnic minority group. An overrepresentation of certain ethnic minority groups under compulsory treatment has previously been reported, ${ }^{31}$ yet controlled observational studies have not found ethnicity to be associated with CTO receipt, after adjusting for associated demographic or clinical factors. ${ }^{15,29}$ Considering the known relationship between ethnicity and psychotic disorders, ${ }^{32}$ it may be that the higher proportion of minority ethnic patients under a CTO reflects the greater prevalence of schizophrenia among the patients subject to a CTO within the NCISH case series. In line with other studies, ${ }^{29,33}$ we also found that comorbid substance misuse was more prevalent among patients subject to a CTO, highlighting the more complex clinical profile of these patients.

\section{Strengths and limitations}

This national study was based on robust case ascertainment and, to our knowledge, was the first to examine suicide rates among patients under a CTO. The findings should, however, be interpreted in light of certain limitations. First, the observational nature of the study precluded elucidation of causal associations. Second, we did not have specific information on risk assessments and the decisionmaking process for patients on a CTO. However, as risk is fundamental to the use of MHA powers, it seems likely that patients subject to its provisions are perceived as being higher risk than those who are not, whether as a result of clinical presentation or non-clinical risk factors. We also cannot establish whether specific interventions to reduce suicide risk were implemented with the patients subject to a CTO, thereby explaining the lower suicide rates. How clinician- or patient-related factors influence a CTO outcome is worthy of further study. Third, the retrospective data collection from clinicians was based on their knowledge of the patient, clinical judgement and case records, as opposed to standardised assessments. However, the reliability and validity of NCISH questionnaire data have been shown to be good, and the NCISH consistently achieves high response rates. ${ }^{20,34}$ Fourth, in patients subject to a CTO, the number of suicides that occurred across the study's observation period indicated an unstable pattern (most likely because of the small number of suicides that occurred each year), and our projected figures for the most recent years may be over- or underestimates. Fifth, denominator data were obtained

Table 4 Suicide rates among patients ever placed on a CTO and among CTO-eligible patients who died more than 12 months after discharge

\begin{tabular}{|c|c|c|c|c|c|c|c|c|c|}
\hline \multirow[b]{2}{*}{ Period of observation, years } & \multicolumn{3}{|c|}{ Ever placed on a Сто } & \multicolumn{3}{|c|}{ Сто-eligible patients } & \multicolumn{3}{|c|}{ Relative risks: IRRS } \\
\hline & $n$ & Rate $^{a}$ & $95 \% \mathrm{Cl}$ & $n$ & Rate $^{\text {b }}$ & $95 \% \mathrm{Cl}$ & IRR & $95 \% \mathrm{Cl}$ & $P$-value \\
\hline 2011-2014 & 27 & 152.7 & $100.7-222.1$ & 100 & 189.5 & $154.2-230.4$ & 0.81 & $0.53-1.17$ & 0.26 \\
\hline $2015-2018$ & 29 & 153.6 & 102.9-220.5 & 120 & 262.6 & $217.8-313.9$ & 0.58 & $0.39-0.84$ & 0.002 \\
\hline Total & 56 & 153.2 & $115.7-198.8$ & 220 & 223.4 & $194.9-254.9$ & 0.69 & $0.52-0.89$ & 0.003 \\
\hline
\end{tabular}


from the MHSDS and therefore were not specifically collected for the purpose of conducting this study. We were therefore unable to stratify the results by age, gender or ethnicity, or estimate suicide rates by duration of care under CTO. Furthermore, some of the aggregate denominators that we applied to calculate rates may have contained multiple episodes; for instance, if more than one CTO had been issued or there were multiple discharges during a year, this may have slightly reduced the accuracy of these calculated rates. Nonetheless, the MHSDS represents the most complete source of national denominator data on mental health activity that is available for academic research in England. Finally, the findings were based on patients in England only and may not be generalisable to the devolved nations of the UK, or to other countries where CTOs are applied. They may also not be applicable to individual healthcare providers where the use of CTOs varies widely. ${ }^{29}$ Part of this variation may lie in differences in case-loads and procedures between healthcare settings, but may also reflect differing experiences of clinicians with regard to CTOs, ${ }^{35}$ which warrants further investigation.

In conclusion, the most important test of CTOs is whether they improve patient safety and lower suicide risk. This is difficult to accomplish via randomised controlled trials for practical and ethical issues, and therefore we are left with conducting prospective observational follow-up studies to further our understanding of the potentially beneficial effect of CTOs. Overall, the findings generated from the current study contribute to the debate as to whether CTOs are effective in enhancing patient safety. The UK Government's 2018 Independent Review of the Mental Health Act $1983^{36}$ considered the implications if CTOs were replaced or reformed. This review concluded that CTOs should remain for the time being, but be reduced in number, whereas their application criteria should be tightened. We have shown evidence of the beneficial effect of CTOs in potentially reducing suicide risk, but further monitoring is needed to determine whether the rates continue to remain lower than among patients who are treated voluntarily. CTOs remain a controversial treatment option and clinicians making judgements about the use of CTOs have to consider not only risk, but factors such as patient autonomy, long-term adherence and potential stigma.

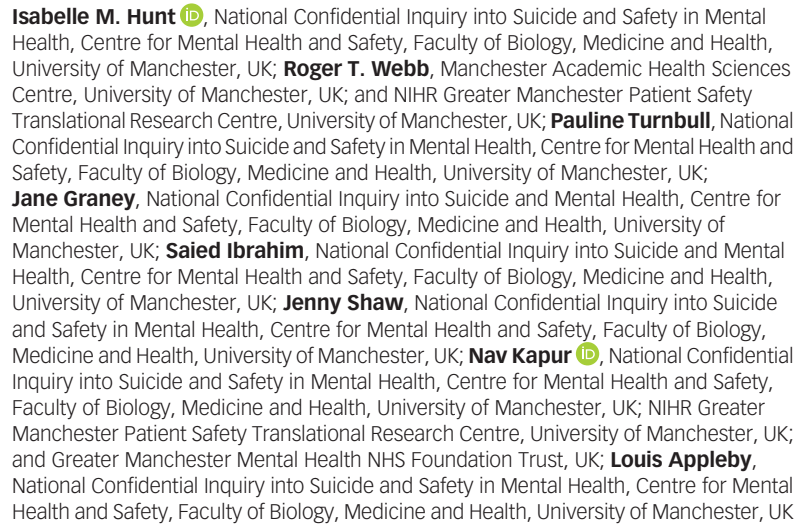

\section{Supplementary material}

Supplementary material is available online at https://doi.org/10.1192/bjo.2021.1021

\section{Data availability}

All authors had full access to all of the data in the study and take responsibility for the integrity of the data and the accuracy of data analysis; access to the data is currently ongoing. Data from this study cannot be shared because of information governance restrictions in place to protect confidentiality. Access to data can be requested via application to the Healthcare Quality Improvement Partnership (www.haip.org.uk/national-programmes/accessing-ncapop-data/).

\section{Acknowledgements}

The study was carried out as part of the National Confidential Inquiry into suicide and Safety in Mental Health (NCISH) and we thank the other members of the research team: Alison Baird, Lana Bojanic, James Burns, Huma Daud, Julie Hall, Rebecca Lowe, Nicola Richards, Cathryn Rodway, Phil Stones and Su-Gwan Tham. We thank the administrative staff in NHS Trusts who helped with the NCISH processes, and the clinicians and nurses who completed the questionnaires.

\section{Author contributions}

All authors were responsible and accountable to all part of works related to the study. I.M.H., P.T., J.G., S.I., J.S., N.K. and L.A. contributed to data acquisition. L.A. conceived the study. I.M.H., R.T.W. and S.I. were responsible for data analysis. L.A., I.M.H., R.T.W., J.G., J.S., P.T. and N.K. were responsible for interpretation of the data. I.M.H. drafted the article under the supervision of R.T.W. N.K. and L.A. All authors critically revised and approved the final manuscript for submission. All authors agree to be accountable for all aspects of the work in ensuring that questions related to the accuracy or integrity of any part of the work are appropriately investigated and resolved.

\section{Funding}

The study was supported by the Healthcare Quality Improvement Partnership (HQIP) and was carried out as part of the NCISH. All researchers are independent from the funders. The funders of the study had no role in study design, data collection and analysis, interpretation of data, preparation of the manuscript or decision to publish. R.T.W. and N.K. are supported by the National Institute for Health Research (NIHR) Greater Manchester Patient Safety Translational Research Centre (grant reference number PSTRC-2016-003). N.K. is also funded by the Greater Manchester Mental Health NHS Foundation Trust.

\section{Declaration of interest}

All authors have completed the ICMJE uniform disclosure form at www.icmie.org/coi disclosure.pdf. L.A. chairs the National Suicide Prevention Strategy Advisory (NSPSA) Group at the Department of Health and Social Care in England (of which N.K. is also a member) and is a non-executive Director for the Care Quality Commission. He was also National Director for Mental Health in England when the CTO was introduced. N.K. chaired the guideline development group for the 2012 National Institute for Health and Clinical Excellence (NICE) guidelines on the longer-term management of self-harm, currently chairs the guideline development group for the NICE depression in adults guidelines and is a topic advisor on the new NICE guideline on self-harm, currently in development. Views expressed in the paper are those of the authors and not those of NICE or the Department of Health and Social Care. All other authors declare no other relationships or activities that could appear to have influenced the submitted work

\section{References}

1 World Health Organization. Suicide Worldwide in 2019: Global Health Estimates. World Health Organization, 2021 (https://www.who.int/publications/i/item/9789240026643).

2 Office for National Statistics. Suicides in England and Wales: 2019 Registrations. Office for National Statistics, 2020 (https://www.ons.gov.uk/peoplepopulationandcommunity/birthsdeathsandmarriages/deaths/bulletins/suicidesintheunitedkingdom/2019registrations).

3 Hawton K, van Heeringen K. Suicide. Lancet 2009; 373(9672): 1372-81.

4 World Health Organization. News Release: The Seventy-fourth World Health Assembly Closes. World Health Organization, 2021 (https://www.who.int/ news/item/31-05-2021-the-seventy-fourth-world-health-assembly-closes).

5 Department of Health. Preventing Suicide in England: A Cross-Government Outcomes Strategy to Save Lives. The Stationery Office, 2012 (https://assets. publishing.service.gov.uk/government/uploads/system/uploads/attach01ment_ data/file/430720/Preventing-Suicide-.pdf)

6 Rugkåsa J, Canvin C. Carer involvement in compulsory out-patient psychiatric care in England. BMC Health Serv Res 2017; 17: 762.

7 Corring D, O'Reilly R, Sommerdyk C. A systematic review of the views and experiences of subjects of community treatment orders. Int J Law Psychiatry 2017; 52: 74-80.

8 Canvin K, Rugkåsa J, Sinclair J, Burns T. Patient, psychiatrist and family carer experiences of community treatment orders: qualitative study. SOC Psychiatry Psychiatr Epidemiol 2014; 49(12): 1873-82.

9 Stroud J, Banks L, Doughty K. Community treatment orders: learning from experiences of service users, practitioners and nearest relatives. I Ment Health 2015; 24(2): 88-92.

10 Munetz MR, Galon PA, Frese FJ 3rd. The ethics of mandatory community treatment. J Am Acad Psychiatry Law 2003; 31: 173-83.

11 McMillan J, Lawn S, Delany-Crowe T. Trust and community treatment orders. Front Psychiatry 2019; 10: 349 
12 Barnett $\mathrm{P}$, Matthews $\mathrm{H}$, Lloyd-Evans B, Mackay E, Pilling S, Johnson S. Compulsory community treatment to reduce readmission to hospital and increase engagement with community care in people with mental illness: a systematic review and meta-analysis. Lancet Psychiatry 2018; 5(12): 1013-22.

13 Burns T, Rugkåsa R, Molodynski A, Dawson J, Yeeles K, Vazquez-Montes $M$ et al. Community treatment orders for patients with psychosis (OCTET): a randomised controlled trial. Lancet 2013; 381(9878): 1627-33.

14 Burns T, Yeeles K, Koshiaris C, Vazquez-Montes M, Molodynski A, Puntis S, et al. Effect of increased compulsion on readmission to hospital or disengagement from community services for patients with psychosis: follow-up of a cohort from the OCTET trial. Lancet Psychiatry 2015; 2: 881-90.

15 Barkhuizen W, Cullen AE, Shetty H, Pritchard M, Stewart R, McGuire P, et al Community treatment orders and associations with readmission rates and duration of psychiatric hospital admission: a controlled electronic case register study. BMJ Open 2020; 10: e035121.

16 weich S, Duncan C, Twigg L, McBride O, Parsons H, Moon G, et al. Use of community treatment orders and their outcomes: an observational study. Health Serv Deliv Res 2020; 8(9): 1-76.

17 National Confidential Inquiry into Suicide and Safety in Mental Health. Annual Report: England, Northern Ireland, Scotland and Wales. University of Manchester, 2019 (https://sites.manchester.ac.uk/ncish/reports/annual-report2019-england-northern-ireland-scotland-and-wales/).

18 Lopez-Morinigo J-D, Ayesa-Arriola R, Torres-Romano B, Fernandes AC, Shetty H, Broadbent $\mathrm{M}$, et al. Risk assessment and suicide by patients with schizophrenia in secondary mental healthcare: a case-control study. BMJ Open 2016; 6: e011929.

19 Phelan JC, Sinkewicz M, Castille DM, Huz S, Link B. Effectiveness and outcomes of assisted outpatient treatment in New York State. Psychiatr Serv 2010; 61(2): 137-43.

20 Appleby L, Shaw J, Amos T, McDonnell R, Harris C, McCann $\mathrm{K}$, et al. Suicide within 12 months of contact with mental health services: national clinical survey. BMJ 1999; 318: 1235-39.

21 Gunnell D, Bennewith O, Simkin S, Cooper J, Klineberg E, Rodway C, et al. Time trends in coroners' use of different verdicts for possible suicides and their impact on officially reported incidence of suicide in England: 1990-2005. Psychol Med 2013; 43(7): 1415-22.

22 NHS Digital. Mental Health Services Data Set. NHS Digital, 2021 (https://digital. nhs.uk/data-and-information/data-collections-and-data-sets/data-sets/mentalhealth-services-data-set)

23 Gardner W, Mulvey EP, Shaw EC. Regression analyses of counts and rates: Poisson, overdispersed Poisson, and negative binomial models. Psychol Bull 1995; 118(3): 392-404.

24 StataCorp. Stata Statistical Software: Release 15. StataCorp LLC, 2017 (https:// www.stata.com/)

25 Chung DT, Ryan CJ, Hadzi-Pavlovic D, Singh SP, Stanton C, Large MM. Suicide rates after discharge from psychiatric facilities: a systematic review and meta-analysis. JAMA Psychiatry 2017; 74(7): 694-702.
26 Haglund A, Lysell $H$, Larsson $H$, Lichtenstein $P$, Runeson B. Suicide immediately after discharge from psychiatric inpatient care: a cohort study of nearly 2.9 million discharges. J Clin Psychiatry 2019; 80(2): 18m12172.

27 Kisely S, Preston N, Xiao J, Lawrence D, Louise S, Crowe E. Reducing all-cause mortality among patients with psychiatric disorders: a population-based study. Can Med Assoc J 2013; 185(1): E50-6.

28 Puntis SR, Rugkåsa J, Burns T. Associations between compulsory community treatment and continuity of care in a three year follow-up of the Oxford Community Treatment Order Trial (OCTET) cohort. BMC Psychiatry 2017; 17: 151

29 Lei H, Barnicot K, Maynard E, Etherington A, Zalewska K, Quirk A, et al. Factors influencing use of community treatment orders and quality of care that people receive: results of a national survey in England and Wales. BJPsych Bull 2019; 43 (5): 227-35

30 Kapur N, Ibrahim S, While D, Baird A, Rodway C, Hunt IM, et al. Mental health service changes, organisational factors, and patient suicide in England in 1997-2012: a before-and-after study. Lancet Psychiatry 2016; 3(6): 526-34.

31 Barnett P, Mackay E, Matthews H, Gate R, Greenwood H, Ariyo K, et al. Ethnic variations in compulsory detention under the Mental Health Act: a systematic review and meta-analysis of international data. Lancet Psychiatry 2019; 6(4): 305-17.

32 Halvorsrud K, Nazroo J, Otis M, Hajdukova EB, Bhui K. Ethnic inequalities in the incidence of diagnosis of severe mental illness in England: a systematic review and new meta-analyses for non-affective and affective psychoses. Soc Psychiatry Psychiatr Epidemiol 2019; 54: 1311-23.

33 Churchill R, Owen G, Singh S, Hotopf M. International Experiences of using Community Treatment Orders. Department of Health and Institute of Psychiatry, King's College London, 2007 (https://webarchive.nationalarchives. gov.uk/20130123191650/http://www.dh.gov.uk/en/Publicationsandstatistics/ Publications/PublicationsPolicyAndGuidance/DH_072730)

34 Appleby L, Shaw J, Kapur N, Windfuhr K, Ashton A, Swinson N, et al. Avoidable Deaths. Five Year Report of the National Confidential Inquiry into Suicide and Homicide by People with Mental Illness. Department of Health, 2006 (http:// documents.manchester.ac.uk/display.aspx?DocID=37602).

35 Manning C, Molodynski A, Rugkåsa J, Dawson J, Burns T. Community treatmen orders in England and Wales: national survey of clinicians' views and use. Psychiatrist 2011; 35: 328-33.

36 Department of Health and Social Care. Modernising the Mental Health Act: Final Report of the Independent Review of the Mental Health Act 1983. The Stationery Office, 2019 (https://www.gov.uk/government/publications/modernising-the-mental-health-act-final-report-from-the-independent-review). 